1. Der Angriff des Glases durch wässrige Säurelösungen ist nicht wesentlich abhängig von der Art und - innerhalb bestimmter Grenzen von der Concentration der Säuren.

2. Der Angriff wässriger Säurelösungen auf Glas erfolgt nur durch das in ihnen enthaltene Wasser.

3. Die Mitwirkung der gelösten Säuren besteht nur darin, das in die Lösung übergehende Alkali zu neutralisiren.

4. Wässrige Säurelösungen greifen Glas schwächer an als reines Wasser.

Ausserdem ergaben sich folgende Sätze:

5. Sehr kalkreiche Gläser sowie bleireichere Flintgläser erfahren durch wässrige Säurelösungen einen sehr starken Angriff, welcher abhängig ist von der Art und Concentration der Säurelösungen.

6. Auf gewöhnliche Kalkgläser wirkt siedende Schwefelsäure schwächer ein als siedendes Wasser.

7. Schwefelsäuredämpfe greifen bei hoher Temperatur das Glas stark an, indem Beschläge von Alkalisulfaten entstehen und bewirken eine tiefgreifende Veränderung der Glasoberfläche.

Charlottenburg, den 25. October 1893 .

\title{
Ceber die Erscheinungen bei der Verwitterung der Gläser und bei der Zersetzung derselben durch Wasser.
}

$$
\text { Von }
$$

\section{F. Foerster.}

(Untersuchung ausgeführt in der Physikalisch-Technischen Reichsanstalt.)

Die Oberflächenveränderungen von Gläsern, welche unter dem Namen der Verwitterungserscheinungen zusammengefasst werden, sind wiederholt Gegenstand der Forschung gewesen, so haben sich Vogel und Reischaue ${ }^{1}$ ), Splittgerber ${ }^{2}$ ), Warburg und Ihmori ${ }^{3}$ ), Kohl-

1) Dingler's polyt. Journ. 152, 181.

2) Dingler's polyt. Journ. 159, 158.

3) Wiedemann's Annalen 27, 481. 
raus ch ${ }^{1}$ ) sowie Mylius und ich ${ }^{2}$ ) mit diesen Erscheinungen beschäftigt, und $R$. Weber ${ }^{3}$ ) hat sie zum Gegenstande besonders eingehender Untersuchungen gemacht. Wenngleich auf Grund der vorliegenden Erfahrungen man schon ein Bild von den Verwitterungsvorgängen gewinnen kann, so erschien es doch erwünscht, noch etwas näher in die Einzelheiten dieser Vorgänge einzudringen. Hierbei schien die Frage, wie weit die Wirkung des Wassers, und wie weit diejenige der Kohlensäure sich erstrecken, angesichts der in der vorhergehenden Abhandlung mitgetheilten Erfahrungen, welche über das Verhalten der Säuren in wässriger Lösung gewonnen wurden, ein gewisses Interesse zu bieten, zumal aus den über das Verhalten der Kohlensäure gegen Glas bisher vorliegenden Arbeiten von Bunsen ${ }^{4}$, Kayser ${ }^{5}$ ) und Krause ${ }^{6}$ ) eine sichere Beantwortung. der Frage nicht möglich ist.

Es handelte sich darum, zu entscheiden, ob die sehr häufig auf verwitterten Glasoberflächen beobachtete Bildung von Alkalicarbonaten einer unmittelbaren Einwirkung der Kohlensäure auf Glas zugeschrieben werden müsse, oder ob diese Salze erst mittelbar aus den durch den Wasserdampf auf dem Glase erzeugten alkalischen Zersetzungsproducten hervorgingen. Diese Frage suchte ich in der Weise zu beantworten, dass ich frische Glaskolben einmal dem Einflusse von Wasserdämpfen, ein anderes Mal unter gleichen äusseren Bedingungen der Wirkung fenchter Kohlensäure aussetzte und die durch diese verschiedene Behandlungsweise hervorgebrachten Aenderungen in der Angreifbarkeit der Kolben durch Wasser bestimmte, als deren Maass, wie bei früheren Versuchen, die in Lösung gehende Menge Alkali diente. Die zu diesen Versuchen angewandten Kolben, der Zahl nach etwa 40, wurden in meiner Gegenwart aus dem gleichen Glashafen in der Hütte der Herren Greiner \& Friedrichs in Stützerbach hergestellt und in einem von. den Verbrennungsgasen der Holzfeuerung durchströmten Kühlofen gekühlt. Unter diesen Umständen verhält sich, wie durch besondere Versuche zuvor ermittelt war, die Oberfäche gekühlter Gläser wie diejenige nicht

1) Wiedemann's Annalen 44, 577 und diese Zeitschrift 31, 421.

2) Ber. d. dentsch. chem. Gesellsch. zu Berlin 22, 1092 und diese Zeitschrift 30, 317 und 31, 241.

3) Dingler's polyt. Journ. 171, 129; Wiedemann's Annalen 6, 431.

4) Wiedemann's Annalen 20, 543; 24, 321; 29, 161.

5) Wiedemann's Annalen 23, 416.

6) Wiedemann's Annalen 36, 923. 
gekühlter, das heisst wie die Glasmasse selbst. Die Kolben wurden während der Herstellung and für die Verpackung sorgfältig vor eindringendem Staube geschützt. Das Glas hatte die Zusammensetzung: $\mathrm{SiO}_{2} 69,4, \mathrm{CaO} 7,0, \mathrm{Al}_{2} \mathrm{O}_{3} 3,0, \mathrm{MnO} 0,4, \mathrm{~K}_{2} \mathrm{O} 7,1, \mathrm{Na}_{2} \mathrm{O}$ 13,1 v. $\mathrm{H}$. Von den ganz frischen Kolben wurden zunächst mehrere Exemplare auf ihre Angreifbarkeit durch Wasser von $20^{\circ}$ von Tag zu Tag untersucht. Von den übrigen Kolben wurden später einige bei $30^{\circ}$ im Strome von Kohlensäure, welche bei Zimmertemperatur mit Wasserdampf gesättigt war, 6 Wochen lang behandelt; andere wurden unter sonst gleichen Bedingungen der Einwirkung von kohlensäurefreier, feuchter Luft (welche also bei $30^{\circ}$ nicht mit Wasserdampf gesättigt war), ausgesetzt. Diese Versuchsanordnung schloss es aus, dass im Innern der Kolben Beschläge flüssigen Wassers auftraten. Nach Beendigung dieser Versuche zeigten die mit Kohlensäure behandelten Kolben in ihrem Innern einen reifartigen, weissen Beschlag von Carbonaten, während die Kolben, welche nur mit Wasserdampf in Berührung gewesen waren, vollkommen ihre Durchsichtigkeit beibehalten hatten. Nunmehr wurden die verschieden behandelten Kolben mit Wasser von $20^{\circ}$ in Berührung gebracht, und zuerst die in der ersten Minute in Lösung übergehenden Alkalimengen bestimmt. Alsdann wurden die Kolben mit neuem Wasser 24 Stunden behandelt, wiederum die in Lösung gegangenen Alkalimengen ermittelt und dies so oft wiederholt, als es nöthig schien. Ausser diesen Versuchen wurden noch einige andere angestellt, welche über die Stärke der Verwitterung unter dem gleichzeitigen Einfluss der atmosphärischen Feuchtigkeit und Kohlensäure Aufschluss geben sollten. Zu diesem Zweck wurden einige Kolben im Zimmer, andere im Freien ein Jahr lang so aufgestellt, dass sie vor hineinfallendem Staube geschützt waren, und dass doch die Luft möglichst frei eintreten kounte. Während dieser Zeit überzog sich die Innenseite der Kolben allmählich mit einem Netzwerk feiner Krystallnadeln, welche im Wesentlichen aus Alkalicarbonaten bestanden. Schliesslich wurden auch noch einige Kolben, welche im Zimmer verwittert waren, 6 Wochen bei $30^{\circ}$ der Einwirkung feuchter Kohlensäure ausgesetzt. Auch bei diesen Versuchsreihen musste der Vergleich der Angreifbarkeit der verwitterten und der frischen Kolben ein Bild von der Grösse der in der Oberfläche vorgegangenen Veränderungen geben.

In der folgenden Uebersicht I sind nicht alle erhaltenen Zahlen angegeben, sondern nur diejenigen, welche für den Angriff der Kolben 
nach der ersten Minute, am 1., 2., 3., 5., 7. und 10. Tage durch Wasser von $20^{\circ}$ ermittelt wurden. Dieselben sind Mittelwerthe aus mehreren Versuchsreihen, bedeuten Tausendstelmilligramme und beziehen sich auf 100 gem Oberfläche.

Uebersicht I.

\begin{tabular}{|c|c|c|c|c|c|c|}
\hline \multirow[b]{2}{*}{$\begin{array}{c}\text { Zeit } \\
\text { der } \\
\text { Behandlung } \\
\text { mit } \\
\text { Wasser }\end{array}$} & \multicolumn{6}{|c|}{ Alkaliabgabe an Wasser von $20^{\circ}$} \\
\hline & $\begin{array}{c}\text { im } \\
\text { frischen } \\
\text { Zustande }\end{array}$ & $\begin{array}{c}\text { nach 6- } \\
\text { wöchent- } \\
\text { licher Be- } \\
\text { handlung } \\
\text { mit } \\
\text { Wasser- } \\
\text { dampf bei } \\
30^{\circ}\end{array}$ & $\begin{array}{c}\text { nach 6- } \\
\text { wöchent- } \\
\text { licher Be- } \\
\text { handlung } \\
\text { mit } \\
\text { feuchter } \\
\text { Kohlen- } \\
\text { säure } \\
\text { bei } 30^{\circ}\end{array}$ & $\begin{array}{l}\text { nach ein- } \\
\text { jähriger } \\
\text { Verwitte- } \\
\text { rung im } \\
\text { Zimmer }\end{array}$ & $\begin{array}{l}\text { nach ein- } \\
\text { jähriger } \\
\text { Verwitte- } \\
\text { rung im } \\
\text { Freien }\end{array}$ & $\begin{array}{l}\text { nach } 1 \text { jähr. } \\
\text { Verwitte- } \\
\text { rung im } \\
\text { Zimmer u. } \\
6 \text { wöchent- } \\
\text { licher Be- } \\
\text { handlung } \\
\text { mitfeuchter } \\
\text { Kohlen- } \\
\text { säure bei } \\
300\end{array}$ \\
\hline in der 1. Min. & 36 & 65 & 59 & 48 & 66 & 109 \\
\hline am 1. Tage & 63 & 62 & 10 & 50 & 45 & 13 \\
\hline am 2. Tage & 19 & - & 7 & 17 & 18 & 6 \\
\hline am 3. Tage & 14 & 17 & 6 & 15 & 15 & 7 \\
\hline am 5. Tage & 14 & 14 & - & 12 & 12 & 6 \\
\hline am 7. Tage & 11 & 9 & - & 9 & 8 & - \\
\hline am 10. Tage & 8 & 8 & 一 & 8 & 8 & 7 \\
\hline
\end{tabular}

Aus diesen Zahlen ersieht man zunächst, dass Wasser auch von der Oberfläche ganz frischer Gläser im ersten Angenblicke des Angriffes sehr beträchtliche Alkalimengen auflöst; es tritt aber sogleich eine sehr schnelle Abnahme des Angriffes ein, denn die nach einem Tage in Lösung gegangene Alkalimenge ist kaum doppelt so gross wie die in der ersten Minute gelöste. Wie bekannt geht nun die Löslichkeit des Glases für die nächste Zeit immer mehr und mehr zurück, bis sie schliesslich, im vorliegenden Falle vom 10. Tage an, längere Zeit nahezu constant bleibt.

Hat nun $30^{\circ}$ warmer Wasserdampf 6 Wochen hindurch auf die Glasoberfläche eingewirkt, so ist dadurch die Menge der in der ersten Minute in Lösung gehenden Alkalien beträchtlich erhöht worden. Trotzdem dadurch eine entsprechende Verminderung der in der äussersten Oberflächenschicht vorhandenen Alkalimengen stattgefunden hat, geht doch bei der folgenden 24-stündigen Behandlung mit Wasser sowie an den weiteren Tagen etwa eben so viel Alkali in Lösung als bei den ganz 
frischen Kolben. Dieser Befund dürfte auf Grund folgender Betrachtungsweise eine Erklärung finden: Wenn Wasserdampf mit Glas in Berührung kommt, so wird er von ihm zunächst chemisch gebunden, indem ein wasserhaltiges Silicat entsteht. Dieses wird bei weiterer Einwirkung des Wasserdampfes immer stärker hydratisirt, bis es schliesslich in Kieselsäurehydrat und freies Alkali zerfällt. Wirkt nun fiüssiges Wasser auf mit Wasserdampf rorbehandelte, also in einem gewissen Stadium der Hydratation befindliche Glasoberflächen ein, so wird ihm zur Herbeiführung der vollkommenen Zersetzung um so weniger zu thun übrig bleiben, je mehr Wasser vorher bereits von der Glassubstanz gebunden war. Von den dadurch entstandenen Hydratationsproducten ist ein Theil noch schwächer wasserhaltig, während ein anderer Theil in der Hydratation schon weiter fortgeschritten ist. Dieser letztere wird schnell und leicht vollkommen zersetzt werden, und daher erscheint der in der ersten Minute erfolgende Angriff des Wassers auf mit Wasserdampf vorbehandelte Glasoberfächen erheblich stärker als anf solche, welche sich in frischen Zustande befinden. Die anderen auf dem Glase noch vorhandenen, wasserärmeren Hydrate brauchen zur völligen Zersetzung eine längere Zeit als jene; sie können daher erst die Menge der später in Lösung gehenden Alkalien vermehren; ihr Vorhandensein vermag die Wirkung aufzuheben, welche die in der ersten Minute erfolgte stärkere Alkalientziehung auf die spätere Angreifbarkeit des Glases sonst ausüben w würde.

Die Hydratationsproducte des Glases bilden den nothwendigen Uebergang zwischen der frischen Glassubstanz und deren schliesslichen, freies Alkali enthaltenden Zersetzangsproducten. Bei der Einwirkung des Wasserdampfes bleibt das Alkali anf dem Glase haften und übt seine wasseranziehende Wirkung aus: es ist, wie Warburg und Ihmori ${ }^{1}$ ) bemerken, die Ursache der mit dem Feuchtigkeitsgehalt der Luft an Stärke wechselnden »temporären Wasserhaut « auf Gläsern. Soweit die Einwirkungsproducte des Wasserdampfes auf Glas nicht gerade aus freiem Alkali bestehen oder ihrer Natur wach diesem sehr nahe kommen, sind sie Träger der erst beim Erhitzen entweichenden »permanenten Wasserhaut $\ll$.

Aus früheren Versuchen wissen wir, dass freies Alkali die Zersetzung der Gläser dürch Wasser zu beschleunigen vermag und zwar

1) Warburg and Ihmori a. a. 0 . 
dadurch, dass es Kieselsäure hydratisirt, diese löst und in Folge dessen das Eindringen des Wassers in die Glasmasse und seine Berührung mit immer neuen Glasschichten erleichtert. An der Hand obiger Anschauungsweise dürfen wir diese Auffassung dahin erweitern, dass das bei der Hydratation des Glases frei werdende Alkali nicht allein die Hydratation der Kieselsäure, sondern auch diejenige der ganzen, überhaupt dazu befähigten Silicate des Glases in hohem Maasse befördert and diese daher ihrer schliesslichen, vollkommenen Zersetzung durch Wasser besonders schnell entgegenführt. Der Angriff der Gläser durch Wasser erfolgt in der Weise, dass nach Aufnahme von Wasser in die Glassubstanz zunächst Alkali in Iösung geht, dies alsdann die Hydratation weiterer Glastheile beschleunigt und damit einerseits auch die Lösung weiteren Alkalis befördert und andererseits durch Aufnahme von Kieselsäure dem Angriff des Wassers neue Wege eröffnet. Diese Wirkung erfolgt um so stärker, je höher die Temperatur und je concentrirter die entstehende Alkalilösung wird, und es findet immer durch die sich lösende Menge von Alkali eine Steigerung der weiter zu lösenden statt. So erklärt es sich, warum die Abnahme der Angreifbarkeit der Gläser so rasch erfolgt, da, je weniger Alkali in der Oberflächenschicht zurückbleibt and dann noch daraus gelöst werden kann, um so schwächer auch seine Rückwirkung auf die Angreifbarkeit weiterer Glastheile werden muss. Die Beschleunigung der Wirkung des Wassers geht von dem sich lösenden Alkali aus, da dasselbe unmittelbar nach seiner Entstehung in Gestalt einer verhältnissmässig concentrirten Lösung die Glasoberfläche ïberzieht. Wenn dàs Alkali ron dieser hinweg in das Wasser hinein diffundirt und sich hier nur in stark verdünnter Lösung befindet, so vermag es die genannte Wirkung auf weitere Glastheile, mit denen es nun in Berührung kommt, nicht mehr auszuüben. Dies zeigt sich dadurch, dass tausendstel-normale Alkalilösungen Glas nicht wesentlich stärker angreifen als reines Wasser. Erst wenn die im Wasser gelösten Alkalimengen immer grössere werden, sei es dass sie aus dem Glase stammen oder voh vorn herein in der Lösung vorhanden waren, machen diese allmählich ihre besondere, früher ${ }^{1}$ ) besprochene Einwirkung auf Glas geltend.

Lassen wir nun die Einwirkung des Wasserdampfes auf Glas bei Gegenwart von Kohlensäure vor sich gehen, so finden wir, dass die in

1) Ber. d. deutsch. chem. Gesellsch. zu Berlin 25, 2505.

Fresenius, Zeitschrift $f$ analyt. Chemie. XXXII. Jahrgang. 
der ersten Minute in Lösung gehenden Alkalimengen fast die gleichen sind wie bei der vorigen Versuchsreihe, dass aber das in den weiteren 24 Stunden abgegebene Alkali seiner Menge nach stark zurückgegangen ist.

Wenn nun im vorliegenden Falle unter dem Einflusse des Wasserdampfes auch freies Alkali entsteht, so wird es doch seine bei den früheren Versuchen ungehindert ausgeübte Wirkang nur schwach zu entfalten vermögen, da es alsbald durch die in grossem Ueberschuss vorhandene Kohlensäure in Bicarbonat verwandelt wird. Es wird also im Ganzen ein schwächerer Angriff : des Wassers auf das Glas erfolgen als im ersten Falle. Dadurch, dass die Kohlensäure einen Theil der wasserreichen Hydrate zersetzt, das entstandene Bicarbonat aber rascher rom Wasser aufgenommen wird als jene hydratisirten Silicate, erscheint die in der ersten Ninute gelöste Alkalimenge hier besonders gross.

Da nun bei dem späteren Angriffe vom Wasser nur wenig Alkali gelöst wird, so kann dieses nicht in dem Maasse, wie es in den beiden ersten Versuchsreihen die grösseren, aus dem Glase in das Wasser übergehenden Mengen freien Alkalis thaten, bei seiner Entstehung wieder hydratisirend auf Glas zurückwirken, und wir erhalten im vorliegenden Falle alsbald die geringe Löslichkeit, welche sich bei den früheren Versuchen erst nach Verlauf von mehreren Tagen einstellte.

Bei der eigentlichen Verwitterung an der Luft, auf welche sich die in Spalte 5 and 6 der Uebersicht I verzeichneten Versuchsreihen beziehen, vereinigt sich die Wirkung des Wasserdampfes mit derjenigen der Kohlensäure, welche hier aber nicht in dem Maasse, wie oben, im Ueberschuss vorhanden ist. Wir sehen denn auch eine Vermehrung des in der ersten Minute gelösten Alkalis gegenüber dem von frischem Glase abgegebenen. Andererseits geht damit eine verhältnissmässig geringe Verminderung der in den ersten 24 Stunden sich lösenden Alkalimengen Hand in Hand, indem die Kohlensäure, deren Einwirkung ja aus der Bildung krystallisirten Carbonates ersichtlich ist, mit der Zeit das entstehende Alkali allmählich immer wieder bindet und so dasselbe an der Ausübung eines Theiles seiner Rückwirkung auf weitere Antheile der Glassubstanz verhindert. Da diese Wirkung aber nur gering ist, ist die am ersten Tage in Lösung gehende Alkalimenge genügend, um bei ihrer Entstehung ihrerseits wieder andere Glastheile zur Lösung: befähigter zu machen. Es verändert also die Verwitterung, soweit sie durch obige Versuche verfolgt wurde, für den späteren Verlauf der Digestion die Löslichkeit des Glases nicht merklich, sondern macht sich 
nur in den allerersten Stadien der Einwirkung des Wassers auf das Glas und auch da nur in geringem Maasse bemerkbar.

Für Fälle sehr starker Verwitterung, wie sie bei sehr schlechten Gläsern oder auch bei weniger schlechten dann vorkommen, wenn letztere etwa im Laufe andauernder Verwitterung mehrfach durch Niederschläge von Wasser benetzt werden, ist natürlich die Veränderung der Oberfläche eine sehr viel stärkere; hier würde auch im Verlaufe einer längeren Behandlung des Glases mit Wasser ihr Einfuss noch erkenubar sein. Es kommt jedoch nur bei feineren Unterschieden in der Angreifbarkeit der Gläser, also bei besseren Glassorten, wesentlich darauf an, dass man den Einfluss der Verwitterung genau in Rechnung zieht, und auf solche Fälle sind die vorstehenden Versuchsergebnisse stets anwendbar.

Lassen wir nun, indem wir die letzte Spalte der Uebersicht $\mathbf{I}$ in Betracht zichen, auf die schon stark verwitterten Gläser noch feuchte Kohlensäure bei $30^{\circ}$ einwirken, so wird diese zunächst sich mit einem Theil der schon vorhandenen Verwitterungsproducte verbinden, einen anderen Theil wird der Wasserdampf für den Angriff durch die Kohlensäure befähigt machen, während er auf die dadurch sehr alkaliarm gewordene Glasoberfläche eine weitere, erhebliche Einwirkung nicht ausüben wird. Es zeigt sich auch hier wieder eine starke Verminderung der nach dem Ausspülen noch verbleibenden, am ersten Tage in Lösung gehenden Alkalimengen, und in Folge dessen nehmen die Mengen des weiter von Tag zu Tag vom Wasser aufgenommenen Alkalis schon anfangs den constanten Werth an, welcher unter anderen Umständen erst später erreicht wird.

Wenn von der Wirkung der Kohlensäure auf die Verwitterungsproducte des Glases die Rede ist, so ist darunter, wie wiederholt schon erwähnt wurde, nicht allein diejenige auf das in jenen vorhandene freie Alkali zu verstehen, sondern je nach der wirkenden Menge der Kohlensäure werden auch mehr oder weniger stark hydratisirte Silicate von ihr zersetzt werden. In jedem Falle muss aber der Einwirkung der Kohlensäure auf das Glas diejenige des Wassers vorhergehen, indem dieses allein das Glas angreift, und die Kohlensäure nur mit den entstandenen Zersetzungsproducten zu reagiren vermag. Eine besondere Wirkung auf Glas konnte für die Kohlensäure eben so wenig nachgewiesen werden wie für andere, bei Gegenwart von Wasser mit Glas in Berührung tretende Säuren. Da auch bei der Verwitterung der in der 
Natur vorkommenden Silicate stets nur feuchte Kohlensäure wirksam ist, so wäre eine vergleichende Untersuchung über das Verhalten der natürlichen Silicate gegen Kohlensäure wohl von Interesse.

Eine sehr werthvolle Bestätigung der soeben mitgetheilten Erfahrungen bilden die Versuche $B$ unsen's ${ }^{1}$ ) über die Wirkung von Kohlensäure auf Glas, besonders weil sie darthum, dass trockene Kohlensäure von trockenem Glas nicht absorbirt wird, also dasselbe nicht angreift. Die übrigen Beobachtungen Bunsen's, welche sich auf das Verhalten von trockener Kohlensäure gegen vorher hydratisirte Glasoberflächen, sowie von Kohlensäure bei Gegenwart beschränkter Mengen von Wasser auf wasserfreies Glas bezogen, finden in den vorangegangenen Darlegungen eine ausreichende Frklärung.

Wenn wir oben zur Erklärung der beobachteten Verwitterungserscheinungen von der Auffassung ansgingen, dass stets die Einwirkung des Wassers damit beginnt, dass eine chemische Bindung von Wasser durch das Glas erfolgt, so liegt darin nur eine Verallgemeinerung längst bekannter Thatsachen. Man weiss, dass, wenn es sich um eine sehr starke Verwitterung handelt, wie solche besonders bei kalireichen Gläsern vorkommt, ohne dass die Glassubstanz ihre äussere Beschaffenheit verändert, von ihr verhältnissmässig grosse Wassermengen aufgenommen werden, welche, wenn sie beim Erhitzen des Glases wieder entweichen, ein Abblättern der Oberflächenschichten veranlassen. Vogel und Reischauer $\mathbf{r}^{1}$ ) haben ebenso wie Splittgerber ${ }^{1}$ ) diese Erscheinung näher studirt, und $\mathrm{R}$. Weber ${ }^{1}$ ) hat sie als Anzeichen vorhandener starker Verwitterung von Glasoberflächen benutzt. Sie tritt nun ebenso wie unter dem Einfluss des Wasserdampfes auch in Folge des Angriffs flüssigen Wassers auf, wie F. Mylius ${ }^{2}$ ) und besonders 0 . Schott ${ }^{3}$ ) näher nachgewiesen haben, und wie wohl Mancher schon im Laboratorium bemerkt hat, wenn er weicheres Glas, welches z. B. in der Spritzflasche oder in einem Kühler andauernd mit Wasser in Berührung gewesen ist, in die Flamme brachte. Wirkt überhitztes. Wasser auf Glas ein, so vermögen, wie manche Versuche der vorangehenden Arbeit ergeben, selbst sehr gute Gläser reichliche Wassermengen in sich aufzunehmen.

1) a. a. 0 .

2) Zeitschrift f. Instrumentenkunde 9,56.

3) Diese Zeitschrift 30, 317 und Zeitschrift f. Instrumentenkunde 9,86. 
Anch der Angriff überhitzten Wasserdampfes führt zu einer so starken Hydratation von Gläsern, dass dadurch häufig deren äussere Beschaffenheit stark verändert wird. Sie überziehen sich dann mit einer weissen Verwitterungsschicht, welche schon kaltem Wasser gegenüber sehr viel angreifbarer ist, als das ursprüngliche Glas. ${ }^{1}$ )

Besonders gut lassen sich die Hydratationserscheinungen bei den Wassergläsern und zum Theil auch bei sehr kalkarmen und alkalireichen Gläsern verfolgen, wie F. Mylius und ich früher ${ }^{2}$ ) gezeigt haben. Wird z. B. Kaliwasserglaspulver mit Wasser zu einem Brei angerührt, so nimmt derselbe unter Wärmeentwickelung schnell an Zühigkeit zu und erhärtet in verhältnissmässig kurzer Zeit zu einer einheitlich erscheinenden, ziemlich harten und elastischen Masse, welche nun durch Wasser viel angreifbarer ist, als das frische Wasserglaspulver. Nimmt man den Versuch mit wenig Wasser in der Hitze vor, so bemerkt man, dass die angewandte Substanz zunächst gelatinirt und alsdann, je nach der angewandten Wasșermenge eine mehr oder weniger viskose Wasserglaslösung bildet. So kann man alle möglichen Zwischenstadien zwischen dem festen Wasserglase und seiner wässrigen Lösung durch geeignete Wahl der Versuchsbedingungen hervorbringen. Wenn viel Wasser auf Wasserglaspulver einwirkt, gelangt das aus demselben frei werdende Alkali in so starke Verdünnung, dass es die Hydratationserscheinungen nicht mehr so stark zu befördern vermag wie unter obigen Bedingungen; dieselben treten daher alsdann auch nicht mehr so dentlich hervor.

Die vom Wasserglase durch die Hydratation aufgenommenen Wassermengen sind verhältnissmässig fest gebunden und entweichen erst bei hoher Temperatur. Das Gleiche gilt für das von Gläsern gebundene Wasser: aus den Versuchen von $\mathrm{Kundt}$ and $\mathrm{Warburg}{ }^{3}$ ) and besonders denen von Bunsen ${ }^{4}$ ) geht hervor, dass mit allmählich ge-

1) Vergi. Ber. d. deutsch. chem. Gesellsch. zu Berlin 25, 2495.

2) Ber. d. deutsch. chem. Gesellsch. zu Berlin 22, 1088 und 1104. Auch eine Beobachtung von Weber und $S$ a uer (Ber. d. deutsch. chem. Gesellsch. zu Berlin 25, 70) gehört hierher, nach welcher ein Kolben aus sehr schlechtem Glase bei andauernder Behandlung mit Wasser von gewöhnlicher Temperatur auf seiner Innenfläche sich mit einer Schicht gallertartiger Zersetzungsproducte bedeckte.

3) Poggendorff's Annalen 156, 201.

4) Wiedemann's Annalen 24, 321 . 
steigerter Temperatur eine immer weitere Wasserabgabe seitens wasserhaltiger Gläser stattfindet, dass diese aber erst bei etwa $500^{\circ} \mathrm{C}$. ein Ende erreicht. Andererseits haben F. Myli us and ich ${ }^{1}$ ) gezeigt, dass man das Abblättern stark verwitterter Gläser schon herbeiführen kann, wenn man diese bei gewöhnlicher Temperatur über Schwefelsäure legt; doch wurde dabei durchaus nicht alles Wasser abgegeben, und die letzten Mengen desselben liessen sich auch erst bei hoher Temperatur austreiben. Diese stetige Wasserabgabe der hydratisirten Gläser ist ein Gegenstück zur Wasseraufuahme, welche wir uns, ähnlich wie es bei den Wassergläsern durch den Versuch verfolgt werden konnte, als eine durchaus stetig erfolgende vorzustellen haben.

Die Wasseraufnahme seitens der Gläser erfolgt nun offenbar nicht nur in solchen Fällen, in denen sie an der Erscheinung des Abblätterns oder durch Wägungen gewöhnlicher Art leicht nachgewiesen werden kann; sondern auch dann, wenn diese Mittel uns nicht mehr erkennen lassen, $o \bar{b}$ eine Hydratation des Glases stattgefunden hat, sind wir berechtigt, eine solche anzunehmen, und zwar um so mehr, als sich, wie die oben gemachten Darlegungen zeigen, die aus dieser Annahme zu ziehenden Folgerungen bewahrheiten. ${ }^{2}$ )

Sowohl bei der Verwitterung wie bei der Zersetzung der Gläser durch Wasser wird dieses vom Glase chemisch gebunden, und es besteht eine Reihe von Uebergängen zwischen der Substanz des Glases und den schliesslich entstehenden Endproducten der Einwirkung des Wassers; bei der Verwitterung bleiben diese in mehr oder weniger veränderter Form auf dem Glase zurück, bei der Zersetzung der Gläser durch Wasser werden sie von diesem mehr oder weniger schnell hinweggeführt. Wir haben es also im letzteren Falle nicht mit einem wirklichen Lösungsvorgange zu thun, bei welchem der Zustand des gelösten Körpers demjenigen des ungelösten unvermittelt gegenübersteht. Hier sowohl. wie bei der Verwitterung liegt vielmehr ein Quellungsvorgang vor, indem unter Betheiligung des Quellungsmittels, des Wassers, der Unterschied zwischen dem festen Glase und seinen Zersetzungsproducten durch eine stetige Reihe von Uebergangssubstanzen überbrückt wird. Ebenso

1) Ber. d. deutsch. chem. Gesellsch. zu Berlin 22, 1109.

2) Auf einer Wasseraufnahme durch die Glassubstanz und nicht auf einer Absorption von Alkali beruhen ganz offenbar gewisse früher (diese Zeitschr. 31, 275) mitgetheilte Erscheinungen, welche hinsichtlich der Angreifbarkeit zuvor andauernd mit Wasser behandelter Gläser beobachtet wurden. 
stêtig findet auch eine gegenseitige Absonderung der bei der Quellung entstehenden Zersetzungsproducte statt, welche schliesslich dahin führt, dass mehr oder minder alkalireiche Silicate in die wässrige Lüsung gehen, während die unlöslichen Producte zurückbleiben.

Der Punkt, bei welchem die Zersetzungsproducte des Glases wirklich gelöst sind, lässt sich nicht sicher bestimmen. Jedenfalls aber kann auch in Alkalisilicatlösungen, welche im Allgemeinen die Eigenschaften der gewöhnlichen Lösungen besitzen, die Reihe der Veränderungen der Alkalisilicate sich noch fortsetzen. So hat $\mathrm{Kohlrausch}{ }^{1}$ ) dargethan, dass in gewissen Lösungen von Alkalisilicaten höchst eigenartige, oft längere Zeit zu ihrer Vollendung brauchende Umwandlungen vor sich gehen können. Schliesslich können auch unter geeigneten Bedingungen aus Alkali- und zumal Natronsilieatlösungen die Silicate sich in krystallisirter Form wieder ausscheiden, worüber Beobachtungen von R. Weber ${ }^{2}$ ) vorliegen.

Wie weit bei den beschriebenen Quellungserscheinungen die alleinige Wirkung des Wassers und wie weit die Beschleunigung derselben durch alkalische Zersetzungsproducte in Frage kommt, lässt sich nicht entscheiden; eben so wenig liegt genügendes Beobachtungsmaterial vor, auf Grund dessen die Veränderungen, welche die Kieselsäure bei jenen Vorgängen erfährt, genauer verfolgt werden könnten.

Sind nun für die Gläser, unter welchen hier wie bei den voraufgehenden Darlegungen zunächst nur die gewöhnlichen Kalkalkaligläser verstanden sein sollen, die Vorgänge bei ihrer Verwitterung und ihrer Zersetzung durch Wasser im Wesentlichen die gleichen, so verwittern sie offenbar um so leichter, je mehr sie vom Wasser angegriffen werden. ${ }^{3}$ ) Obgleich der nahe Zusammenhang beider Erscheinungen bisher wohl nicht vollkommen erkannt war, hat man ihn doch fast allgemein als selbstrerständlich angenommen, und wiederholt ist er in Experimentaluntersuchungen zu Tage getreten. So konnte Weber seine auf Grund

1) F. Kohlrausch, Nachrichten d. königl. Gesellsch. d. Wissensch, zu Göttingen, 1892, S. 461 und Zeitschr. f. physik. Chemie 12, 773.

2) Centralzeitung f. Optik u. Mechanik 1888, S. 258 u. Sprechsaal 1891, S. 589.

3) $0 b$ die Uebereinstimmung im Wesen beider Vorgänge eine alle Einzelheiten umfassende, ganz vollkommene ist, kann zunächst noch nicht mit Sicherheit angegeben werden. Auch muss betont werden, dass alle oben mitgetheilten Exfahrungen sich auf die gewöhnlichen Kalkgläser beziehen, und dass entsprechende Beobachtungen über Bleigläser bisher nicht vorliegen. 
des Verhaltens der Gläser gegen Wasserdampf gewonnenen Erfahrungen erfolgreich zur Beurtheilung der Gläser hinsichtlich ibrer Widerstandsfähigkeit gegen flüssiges Wasser und wässrige Lösungen verwerthen, und so ergab sich stets die gleiche Reihenfolge der Gläser, ob sie nach der die Verwitterungsfähigkeit anzeigenden Weber'schen Probe oder nach den die Angreifbarkeit der Gläser durch Wasser kennzeichnenden Methoden von Mylius und mir ${ }^{1}$ ) und von Kohlrausch ${ }^{1}$ gepruft wurden. Durch einen unmittelbaren Vergleich zeigte auch Kohlrausch, dass diejenigen Gläser am meisten Wasser auf ihrer Oberfläche niederschlagen, welche am leichtesten von Wasser gelöst werden.

Aehnliches konnte ich für eine Anzahl Gläser ebenfalls nachweisen; andererseits liess sich durch den Versuch auch feststellen, dass die aus der Analogie der Verwitterungs- und der Lösungserscheinungen zu ziehende Folgerung richtig sei, dass eine Behandlung von Gläsern mit Wasser eine darauf folgende Verwitterung eben so wenig ausschliesst, wie sie die weitere Löslichkeit aufhebt, dass also die Verwitterungserscheinungen nicht an das Vorhandensein frischer Glasoberflächen gebunden sind. Die bei diesen Versuchsreihen erhaltenen Zahlen können hier der Kürze wegen um so eher übergangen werden, als die genannte Thatsache für sehr schlechte Gläser bereits wiederholt ${ }^{2}$ ) beobachtet wurde.

Da die Verwitterungserscheinungen daher rühren, dass Wasserdampf das Glas zersetzt und in den Zersetzungsprodacten gebunden wird, also sich auf Glas niederschlägt, und es andererseits nicht wahrscheinlich ist, dass Wasser oder Wasserdampf, von meteorologischen Niederschlägen abgesehen, auf anderem als chemischem Wege in merklicher Menge auf Glasoberflächen festgehalten wird, so kann zur Zeit zwischen der Verwitterungsfähigkeit der Gläser und ihrer Hygroskopicität kein Unterschied gefunden werden.

Durch die vorstehenden und früheren den gleichen Gegenstand betreffenden Untersuchungen dürfen folgende Sätze als festgestellt gelten:

1) Die Verwitterungserscheinungen an Gläsern werden durch die zersetzende Wirkung des atmosphärischen Wasserdampfes bedingt; die Kohlensäure der Luft wirkt nicht unmittelbar auf das Glas ein, sondern

1) a. a. 0 .

2) R. Weber, Dingler's polyt. Journ. 171, 129 und Siegwart, Dingler's polyt. Journ. 205, 51 . 
nur auf die alkalischen, durch die Verwitterung auf dem Glase erzeugten Zersetzungsproducte.

2) Trockene Kohlensäure wirkt auf wasserfreies Glas nicht ein.

3) Es ist bisher kein Beweis dafür erbracht, dass Wasser vom Glase anders als auf Grund chemischer Anziehung festgehalten werden kann.

4) Der Verwitterungsvorgang und der Vorgang bei der Zersetzung der Gläser durch Wasser sind ihrem Wesen nach als gleichartig und zwar als Quellungsvorgänge zu betrachten.

5) Die durch die Verwitterung verursachten Veränderungen von Glasoberflächen sind bei besseren Gläsern verhältnissmässig gering.

6) Der Angriff des Wassers auf verwitterte Gläser ist im Allgemeinen nur während der ersten Zeit der Einwirkung des Wassers stärker als derjenige, welcher unter gleichen Bedingungen auf frische Gläser erfolgt.

7) Gläser (Kalkgläser) sind um so hygroskopischer und verwittern um so leichter, je mehr sie rom Wasser angegriffen werden.

8) Auch nach längerer Einwirkung des Wassers auf Glas vermag dieses noch zu verwittern.

Ueber die quantitative Bestimmung der gewöhnlichsten Beimischungen des im Handel vorkommenden Reinnickels oder Walznickels.

Von

Dr. Th. Fleitmann.

Die gewöhnlichsten Beimischungen oder Unreinigkeiten, die sich im walzbaren Nickel finden, sind, sofern dasselbe nach meinem Verfahren (Magnesiumzusatz) hergestellt worden ist, Eisen, Kupfer, Kobalt und geringe Mengen Zink, Bestandtheile, die dem Rohnickel (Würfel oder Pulvernickel) entstammen, aus welchem das Walznickel geschmolzen worden ist. Ist dasselbe nach einem der anderen Verfahren erzeugt worden, welche später dem meinigen nachgebildet wurden, so erhält das Walznickel häufig noch erhebliche Mengen Mangan neben den oben genannten Metallen.

Die quantitative Bestimmung sämmtlicher 5 Beimischungen lässt sich nach meinen Erfahrungen am raschesten und leichtesten in der folgenden Weise ausführen : 